\title{
Special ramification loci on the double product of a general curve *
}

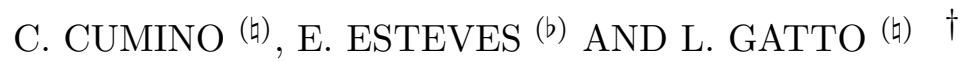 \\ (घ) Dipartimento di Matematica, Politecnico di Torino, \\ Corso Duca degli Abruzzi 24, 10129 Torino - (ITALY) \\ (b) Instituto Nacional de Matemática Pura e Aplicada, \\ Estrada Dona Castorina 110
} 22460-320 Rio de Janeiro RJ - (BRAZIL)

\begin{abstract}
Let $C$ be a general connected, smooth, projective curve of positive genus $g$. For each integer $i \geq 0$ we give formulas for the number of pairs $(P, Q) \in C \times C$ off the diagonal such that $(g+i-1) Q-(i+1) P$ is linearly equivalent to an effective divisor, and the number of pairs $(P, Q) \in C \times C$ off the diagonal such that $(g+i+1) Q-(i+1) P$ is linearly equivalent to a moving effective divisor.
\end{abstract}

\section{Introduction}

Let $C$ be a general connected, smooth, projective curve of genus $g>0$. Put $C^{2}:=C \times C$, and let $\Delta \subset C^{2}$ be the diagonal. For each integer $i \geq 0$ consider

*2000 Mathematics Subject Classification: 14H10, 14H15, 14 N10.

†Work partially sponsored by MURST (Progetto Nazionale "Geometria delle Varietà Proiettive" Coordinatore Sandro Verra), and supported by GNSAGA-INDAM. The second author was also supported by CNPq, Proc. 478625/03-0 and 301117/04-7, and CNPq/FAPERJ, Proc. E-26/171.174/2003. 
the following loci on $C^{2}$ :

$$
\begin{aligned}
D_{i} & :=\left\{(P, Q) \in C^{2}-\Delta \mid h^{0}\left(\mathcal{O}_{C}((g+i-1) Q-(i+1) P)\right)>0\right\} \\
E_{i} & :=\left\{(P, Q) \in C^{2}-\Delta \mid h^{0}\left(\mathcal{O}_{C}((g+i+1) Q-(i+1) P)\right)>1\right\} .
\end{aligned}
$$

Our Proposition 5.4 claims that $D_{i}$ and $E_{i}$ are finite, and our main result, Theorem 5.6, gives formulas for the number of points in $D_{i}$ and $E_{i}$.

A formula for the number of points in $D_{i}$ appeared already as Lemma 6.3 on page 24 of the seminal work by Diaz [6], where the unnecessary extra hypotheses that $g \geq 2$ and $i \geq 2$ are made. Diaz used this formula to compute the class in the moduli space of genus- $g$ stable curves $\overline{\mathcal{M}}_{g}$ of the closure $\overline{\mathcal{D}}_{g}$ of the locus of smooth curves $C$ having a Weierstrass point $P$ of type $g-1$, i.e. such that $h^{0}\left(\mathcal{O}_{C}((g-1) P)\right) \geq 2$.

Later on, Cukierman [4] gave a formula for the class in $\overline{\mathcal{M}}_{g}$ of the closure $\overline{\mathcal{E}}_{g}$ of the locus of smooth curves $C$ containing a Weierstrass point $P$ of type $g+1$, i.e. such that $h^{0}\left(\mathcal{O}_{C}((g+1) P)\right) \geq 3$. He did not follow in Diaz's footsteps for this formula, but rather observed that the union $\overline{\mathcal{D}}_{g} \cup \overline{\mathcal{E}}_{g}$ is the branch locus of the Weierstrass divisor on the "universal" curve over $\overline{\mathcal{M}}_{g}$, and used a Hurwitz formula with singularities to compute the class of this branch divisor.

Had Cukierman followed in Diaz's footsteps, he would probably have found he needed a formula for the number of points in $E_{i}$. We give this formula here.

In fact, in a sense to be explained below, it is slightly easier to obtain the number of points in $E_{i}$ than in $D_{i}$, though we obtain both in a quite integrated form here. To obtain these numbers, the natural procedure is to use Porteous formula to compute the virtual classes of certain natural ramification schemes $D_{i}^{+}$and $E_{i}^{+}$of maps of vector bundles on $C^{2}$; see Subsection 5.2 . Set-theoretically, $D_{i}^{+}$and $E_{i}^{+}$are given exactly as $D_{i}$ and $E_{i}$, but without the restriction that the pair $(P, Q)$ lies off $\Delta$.

The problem is that $D_{i}^{+}$and $E_{i}^{+}$are both larger than $D_{i}$ and $E_{i}$. Indeed, $E_{i}^{+}$is the union of $E_{i}$ with the set of points $(P, P)$ such that $P$ is a Weierstrass point of $C$ and, worse, $D_{i}^{+}$is the union of $D_{i}$ and the whole diagonal $\Delta$. Since $E_{i}^{+}$is finite, Porteous formula does give an expression for the number of points in $E_{i}^{+}$, with weights, and thus at least an upper bound for the number of points in $E_{i}$. But it does not a priori give any information on $D_{i}$.

To compute the number of points in $D_{i}$ and $E_{i}$, we use the fact that, by the Riemann-Roch Theorem, the union of $D_{i}$ and $E_{i}$ is the locus $S W_{i}$ of pairs 
$(P, Q) \in C^{2}-\Delta$ such that $Q$ is a special ramification point of the complete linear system $H^{0}\left(\omega_{C}((i+1) P)\right)$, where $\omega_{C}$ is the canonical bundle of $C$.

We give $S W_{i}$ a scheme structure as follows. First, we consider the ramification divisor $Z_{i} \subset C^{2}$ of the family of linear systems $H^{0}\left(\omega_{C}((i+1) P)\right)$ parameterized by $P \in C$. Our Proposition 2.2 implies that $Z_{i}$ contains $\Delta$ with multiplicity exactly $g$. Set $W_{i}:=Z_{i}-g \Delta$. Our Proposition 4.3 gives an expression for the cycle $\left[W_{i}\right]$, and our Proposition 4.4 claims that $W_{i}$ is nonsingular. Furthermore, in Subsection 5.2 we observe that the branch divisor of $W_{i}$ with respect to the projection $p_{1}: C^{2} \rightarrow C$ over the first factor has support $S W_{i}$. We give $S W_{i}$ the structure of this branch divisor.

The advantage of considering $S W_{i}$ is that it is quite easy to compute its degree. Indeed, $\left[S W_{i}\right]$ is the second Chern class of the bundle of first-order relative jets of $p_{1}$ with coefficients in $\mathcal{O}_{C^{2}}\left(W_{i}\right)$. Having an expression for $\left[W_{i}\right]$ we derive very quickly an expression for $\int_{C^{2}}\left[S W_{i}\right]$ in Proposition [5.5

Now, giving $D_{i}$ and $E_{i}$ the subscheme structures induced from $D_{i}^{+}$and $E_{i}^{+}$, our Proposition 5.4 shows that, as 0-cycles,

$$
\left[D_{i}\right]+\left[E_{i}\right]=\left[S W_{i}\right] .
$$

Actually, $D_{i}$ and $E_{i}$ are reduced. Indeed, in the proof of Theorem 5.6 we show that the weight of $(P, Q)$ in $\left[S W_{i}\right]$ is at most 2, and the maximum weight is achieved if and only if $(P, Q) \in D_{i} \cap E_{i}$.

Now, as we already know $\int_{C^{2}}\left[S W_{i}\right]$, it is enough to compute either $\int_{C^{2}}\left[D_{i}\right]$ or $\int_{C^{2}}\left[E_{i}\right]$. As mentioned above, we compute the latter. In fact, we can get $\int_{C^{2}}\left[E_{i}^{+}\right]$using Porteous formula, and a local analysis, done in Proposition 5.4, shows that the weight of $(P, P)$ in $\left[E_{i}^{+}\right]$is equal to $g+1$ for each Weierstrass point of $C$. Thus $\int_{C^{2}}\left[E_{i}\right]$ follows.

In a second article [5], we show how the knowledge of the number of points in $E_{i}$ can be used to compute the class of $\overline{\mathcal{E}}_{g}$ in $\overline{\mathcal{M}}_{g}$. This computation is not straightforward as, following in Diaz's footsteps, we have to determine the limits of special Weierstrass points of type $g+1$ on stable curves with just one node. This is the main result of [5].

The limits of special Weierstrass points of type $g-1$ were computed by Diaz, using admissible covers. However, the same method does not apply to points of type $g+1$. For those we apply in [5] the theory of limit linear series in a rather new way, using 2-parameter families. Actually, as in the present article, we use an integrated approach in [5] that yields simultaneously the limits of special Weierstrass points of both types, and also formulas for the classes of both $\overline{\mathcal{D}}_{g}$ and $\overline{\mathcal{E}}_{g}$. 
Here is a layout of the article. In Section 2 we review the theory of linear systems and ramification on a smooth curve $C$, introduce the linear systems we will consider in the remaining of the article, $H^{0}\left(\omega_{C}((i+1) P)\right)$ for $P \in C$, and prove a preliminary result about them. In Section 3 , assuming $C$ is general, we obtain through degeneration methods results that bound the order sequence of $H^{0}\left(\omega_{C}((i+1) P)\right)$ at any point of $C$. In Section 4, we describe the structure of the ramification divisor $Z_{i} \subset C^{2}$ of the family of linear systems $H^{0}\left(\omega_{C}((i+1) P)\right)$ parameterized by $P \in C$. Finally, in Section 5 we define the loci $D_{i}$ and $E_{i}$ and compute their number of points, through the study of the locus $S W_{i}$ of special ramification points of the family $H^{0}\left(\omega_{C}((i+1) P)\right)$ for $P \in C$.

We thank Nivaldo Medeiros for discussions on related topics. Also, we acknowledge the use of $\mathrm{CoCoA}[3]$ for some of the computations.

\section{Setup}

2.1 (Linear systems and ramification) Let $C$ be a smooth curve, that is, a projective, connected, smooth scheme of dimension 1 over $\mathbb{C}$. Denote by $\omega_{C}$ its canonical sheaf. Let $g:=h^{0}\left(C, \omega_{C}\right)$, the genus of $C$.

Let $V$ be a $\mathbb{C}$-vector space of sections of a line bundle $\mathcal{L}$ on $C$. We call $V$ a linear system. The linear system is called complete if $V=H^{0}(C, \mathcal{L})$. Let $r:=\operatorname{dim} V-1$ and $d:=\operatorname{deg} \mathcal{L}$. We call $r$ the rank of $V$ and $d$ its degree. We say as well that $\operatorname{dim} V$ is the dimension of $V$.

For each point $P$ of $C$, and each integer $j \geq 0$, let $V(-j P)$ denote the vector subspace of $V$ of sections of $\mathcal{L}$ that vanish with order at least $j$ at $P$. We say that $j$ is an order of $V$ at $P$ if $V(-j P) \neq V(-(j+1) P)$. There are $r+1$ orders, which, in an increasing sequence, will be denoted by

$$
\epsilon_{0}(V, P), \epsilon_{1}(V, P), \ldots, \epsilon_{r}(V, P) .
$$

For each integer $\ell \geq 0$ and each line bundle $\mathcal{M}$ on $C$, let $\mathcal{J}_{C}^{\ell}(\mathcal{M})$ be the bundle of jets, or principal parts, of $\mathcal{M}$ truncated in order $\ell$. Consider the map of rank-r bundles,

$$
V \otimes \mathcal{O}_{C} \longrightarrow \mathcal{J}_{C}^{r}(\mathcal{L})
$$

locally obtained by differentiating up to order $r$ the sections of $\mathcal{L}$ in $V$. The wronskian $w_{V}$ of $V$ is the (nonzero) section of

$$
\mathcal{L}^{\otimes r+1} \otimes \omega_{C}^{\otimes r(r+1) / 2}
$$


induced by taking determinants in the above map of bundles.

For each point $P$ of $C$, the weight $\mathrm{wt}_{V}(P)$ of $P$ in $V$ is the order of vanishing of $w_{V}$ at $P$. We call $P$ a ramification point of $V$ if $\operatorname{wt}_{V}(P)>0$; otherwise we call $P$ ordinary. A local analysis yields the formula

$$
\mathrm{wt}_{V}(P)=\sum_{j=0}^{r}\left(\epsilon_{j}(V, P)-j\right) .
$$

We call $P$ a simple ramification point if $\operatorname{wt}_{V}(P)=1$; otherwise we call $P$ special. The point $P$ is special if and only if the section

$$
D w_{V} \in H^{0}\left(C, \mathcal{J}_{C}^{1}\left(\mathcal{L}^{\otimes r+1} \otimes \omega_{C}^{\otimes r(r+1) / 2}\right)\right),
$$

locally obtained from $w_{V}$ by differentiating, vanishes at $P$.

The total weight of the ramification points of $V$ is the (finite) sum

$$
\mathrm{wt}_{V}:=\sum_{P \in C} \mathrm{wt}_{V}(P)
$$

It is equal to the degree of the line bundle of which $w_{V}$ is a section, that is,

$$
\mathrm{wt}_{V}=(r+1)(d+(g-1) r)
$$

a formula usually referred to as the Brill-Segre or Plücker formula.

The canonical system is the complete linear system of sections of $\omega_{C}$. Its rank is $g-1$, and its degree is $2(g-1)$. For each point $P$ of $C$, its Weierstrass weight $\mathrm{wt}(P)$ is its weight in the canonical system, and the Weierstrass order sequence at $P$ is the increasing sequence of orders at $P$ of the canonical system.

For each integer $i \geq-1$ and each $P \in C$, let $V_{C}(i, P)$ denote the complete linear system of sections of $\omega_{C}((i+1) P)$.

2.2 Proposition. Let $C$ be a smooth curve of genus $g$. For each integer $i \geq 0$ and each $P \in C$, the following two statements hold for $V:=V_{C}(i, P)$ :

1. The weight $\mathrm{wt}_{V}(P)$ of $P$ as a ramification point of $V$ satisfies

$$
\mathrm{wt}_{V}(P)=g+\mathrm{wt}(P)
$$

where $\operatorname{wt}(P)$ is the Weierstrass weight of $P$. 
2. The total weight $\mathrm{wt}_{V}$ of the ramification points of $V$ satisfies

$$
\mathrm{wt}_{V}=g(g+i)^{2} .
$$

Proof. From the Riemann-Roch theorem, for each $j=0, \ldots, i$,

$$
\operatorname{dim} V(-j P)=g+i-j .
$$

In particular, comparing dimensions, we get

$$
V(-i P)=V(-(i+1) P)=H^{0}\left(C, \omega_{C}\right) .
$$

Hence, the order sequence of $V$ at $P$ is

$$
0,1, \ldots, i-1,(i+1)+\epsilon_{0},(i+1)+\epsilon_{1}, \ldots,(i+1)+\epsilon_{g-1},
$$

where $\epsilon_{0}, \epsilon_{1}, \ldots, \epsilon_{g-1}$ is the Weierstrass order sequence at $P$. Thus

$$
\mathrm{wt}_{V}(P)=\sum_{k=0}^{g-1}\left(i+1+\epsilon_{k}-i-k\right)=g+\mathrm{wt}(P) .
$$

The second statement is a direct application of the Brill-Segre formula, using that the rank of $V$ is $g+i-1$ and its degree is $2 g-2+(i+1)$.

\section{The general curve}

3.1 Proposition. Fix an integer $i_{0} \geq 0$. Let $C$ be a general smooth curve of genus $g \geq 1$. Then the following two statements hold for each nonnegative integer $i \leq i_{0}$ :

1. For a general point $P$ of $C$, the linear system $V_{C}(i, P)$ ramifies at $P$ with weight $g$, and has otherwise at most simple ramification points.

2. For any two points $P$ and $R$ of $C$,

$$
\begin{gathered}
h^{0}\left(C, \omega_{C}((i+1) P-(g+i) R)\right) \leq 1 \\
h^{0}\left(C, \omega_{C}((i+1) P-(g+i+2) R)=0 .\right.
\end{gathered}
$$


Proof. Let us first observe that the property required of $C$ is open. Indeed, let $f: X \rightarrow S$ be any family of smooth curves, that is, a projective, smooth map with connected fibers of dimension 1 . Consider the fibered product $X^{(2)}:=X \times_{S} X$ of two copies of $f$, and denote by $p_{1}$ and $p_{2}$ the projection maps. Denote by $\Delta$ the diagonal subscheme of $X^{(2)}$. Let $\omega_{f}$ denote the relative canonical bundle of $f$. Then $\omega:=p_{2}^{*} \omega_{f}$ is the relative canonical bundle of $p_{1}$. Let

$$
\mathcal{V}:=p_{1 *}(\omega((i+1) \Delta))
$$

A fiberwise analysis shows that $\mathcal{V}$ is a bundle of rank $g+i$ with formation commuting with base change. For each integer $\ell \geq 0$, denote by $\mathcal{J}^{\ell}$ the bundle of rank $\ell+1$ of $p_{1}$-relative jets of $\omega((i+1) \Delta)$ truncated in order $\ell$, and denote by $\psi_{\ell}: p_{1}^{*} \mathcal{V} \rightarrow \mathcal{J}^{\ell}$ the map locally obtained by differentiating the sections of $\omega((i+1) \Delta)$ up to order $\ell$ along the fibers of $p_{1}$. Let $W_{i, 1}$ (resp. $\left.W_{i, 2}\right)$ be the closed subset of $X^{(2)}$ where $\psi_{g+i-1}\left(\right.$ resp. $\left.\psi_{g+i+1}\right)$ has rank at most $g+i-2$ (resp. $g+i-1)$. Also, let $W_{i}$ be the closed subset of $X^{(2)}$ where $\psi_{g+i-1}$ has rank at most $g+i-1$. By Proposition 2.2 , $W_{i}$ contains $\Delta$ with multiplicity $g$. Let $W_{i}^{\prime}:=W_{i}-g \Delta$ and $Z_{i}:=\Delta \cap W_{i}^{\prime}$. Let $W_{i}^{\prime \prime} \subset W_{i}^{\prime}$ be the ramification scheme of the map $\left.p_{1}\right|_{W_{i}^{\prime}}$. Let $U_{i} \subseteq S$ be the intersection of $S-f\left(p_{1}\left(W_{i, 1} \cup W_{i, 2}\right)\right)$ with $f\left(X-p_{1}\left(W_{i}^{\prime \prime} \cup Z_{i}\right)\right)$. Since $p_{1}$ is proper, and $f$ is both proper and open, $U_{i}$ is an open subscheme of $S$. Let $U:=U_{0} \cap \cdots \cap U_{i_{0}}$. The formation of $U$ commutes with base change. Thus a fiberwise analysis reveals that $U$ consists of the set of points $s \in S$ such that the proposition holds for $C:=X(s)$.

Now, keeping in mind the existence of a versal family of smooth curves, it is enough to exhibit a single curve $C$ for which the statement holds. We will actually show a somewhat stronger existence result:

3.2 Lemma. Fix nonnegative integers $i_{0}$ and $j_{0}$. Let $g$ be a positive integer. Then there is a smooth pointed curve $(C, Q)$ of genus $g$ for which the following three statements hold for each nonnegative integers $i \leq i_{0}$ and $j \leq j_{0}$ :

1. The linear system $V_{C}(j, Q)$ ramifies at $Q$ with weight $g$, and has otherwise at most simple ramification points.

2. For each $P \in C$ distinct from $Q$, either $Q$ is an ordinary point or a simple ramification point of $V_{C}(i, P)$.

3. For each $P \in C$ distinct from $Q$, the linear system $V$ of sections of 


$$
\begin{aligned}
& \omega_{C}((i+1) P+(j+1) Q) \text { given by } \\
& \qquad:=H^{0}\left(\omega_{C}((i+1) P)\right)+H^{0}\left(\omega_{C}((j+1) Q)\right)
\end{aligned}
$$

satisfies

$$
\operatorname{dim} V(-(g+i+j) R) \leq 1 \quad \text { and } \quad V(-(g+i+j+2) R)=0
$$

for each $R \in C$ distinct from $P$ and $Q$.

We will first see how the lemma implies the proposition. Set $j_{0}=i_{0}$, and consider the pointed curve $(C, Q)$ given by the lemma. Then the two statements of Proposition 3.1 hold for $C$. Indeed, the first statement holds for $P=Q$, whence for $P$ in a neighborhood of $Q$, that is, for a general $P$.

As for the second statement, first notice that (3) and (4) hold for $P=Q$ and every $R \in C$, a consequence of the first statement of the lemma for $j:=i$. They hold as well for $R=Q$ and every $P \in C$ distinct from $Q$, a consequence of the second statement of the lemma. Furthermore, they hold for $R=P$ and any $P \in C$. Indeed, the first statement of the lemma for $j:=0$ implies that the canonical linear system has at most simple ramification points. Thus $h^{0}\left(\omega_{C}((1-g) P)\right) \leq 1$ and $h^{0}\left(\omega_{C}(-(g+1) P)\right)=0$.

Finally, fix a point $P \in C$ distinct from $Q$ and a point $R \in C$ distinct from $P$ and $Q$. For $j:=0$, the linear system $V$ defined in the lemma is the system of sections of $\omega_{C}((i+1) P+Q)$ that are zero on $Q$. Since $R \neq Q$, the third statement of the lemma yields (3) and (4).

It is thus enough to prove the lemma, what we do below.

Proof. (Lemma 3.2) We will do induction on $g$. The initial step is taken care of below.

Let $C$ be any elliptic curve and $Q \in C$ any point. Then the ramification points of the complete linear system of sections of $\omega_{C}((j+1) Q)$ are simple. (These are the $(j+1)^{2}$ points $R$ for which $Q-R$ is $(j+1)$-torsion, what includes $Q$.) In fact, it follows from the Riemann-Roch theorem that every complete linear system has only simple ramification points. Thus Statements 1 and 2 of the lemma hold. Now, given $P \in C$ distinct from $Q$, since the vector subspace $V$ of $H^{0}\left(\omega_{C}((i+1) P+(j+1) Q)\right)$ defined in Statement 3 has codimension 1, the order sequence of $V$ at a point $R$ is obtained either from

$0,1, \ldots, g+i+j-1, g+i+j \quad$ or $\quad 0,1, \ldots, g+i+j-1, g+i+j+1$ 
by removing an order. In any case, there is at most one order of $V$ at $R$ above $g+i+j-1$, that is $\operatorname{dim} V(-(g+i+j) R) \leq 1$, and all orders are at most $g+i+j+1$, that is $V(-(g+i+j+2) R)=0$.

Assume from now on that $g>1$, and that the claim holds for smaller genera and any integers $i_{0}$ and $j_{0}$. We will employ a degeneration technique in order to apply the induction hypothesis.

Let $(Y, A)$ and $(Z, B)$ be nonrational smooth pointed curves of genera $g_{Y}$ and $g_{Z}$, with $g_{Y}+g_{Z}=g$. From the induction hypothesis, we may assume that the statements of the lemma hold for $(C, Q)$ replaced by $(Y, A)$ and all nonnegative integers $i \leq i_{0}$ and $j \leq g_{Z}+i_{0}+j_{0}+1$, and for $(C, Q)$ replaced by $(Z, B)$ and all nonnegative integers $i \leq i_{0}$ and $j \leq g_{Y}+i_{0}+j_{0}+1$.

Let $C_{0}$ be the curve of compact type that is the union of $Y$, of $Z$, and of a chain of rational curves $E_{1}, \ldots, E_{n-1}$ connecting $A$ to $B$, where $n \geq 2$. Our convention is that $E_{1}$ contains $A$ and $E_{n-1}$ contains $B$. Let $v$ be any integer such that $0<v<n$, and let $Q_{0}$ be any point of $E_{v}$ that is not a node of $C_{0}$.

Let $S:=\operatorname{Spec}(\mathbb{C}[[t]])$, and denote its special point by 0 and generic point by $\eta$. Since there are no obstructions to deforming pointed nodal curves, there are a projective, flat map $f: X \rightarrow S$ and a section $\lambda: S \rightarrow X$ of $f$ such that $(X(0), \lambda(0))=\left(C_{0}, Q_{0}\right)$ and $(X(\eta), \lambda(\eta))$ is a smooth pointed curve over the field of formal Laurent series $\mathbb{C}[[t]][1 / t]$.

Let $C$ be the base extension of $X(\eta)$ to the algebraic closure of $\mathbb{C}[[t]][1 / t]$. Set $Q:=\lambda(\eta)$. It is enough to see that the statements of the lemma hold for $(C, Q)$. Indeed, the argument is quite standard, and is summarized below. Though the pointed curve $(C, Q)$ is not defined over $\mathbb{C}$, it is defined over a finitely generated extension $L$ of $\mathbb{Q}$. If the statements of the lemma hold for $(C, Q)$, they also hold for the base extension of $(C, Q)$ over any algebraically closed field containing $L$. But, since $\mathbb{C}$ has many transcendentals over $\mathbb{Q}$, there is an algebraically closed field containing $L$ which is isomorphic to $\mathbb{C}$. So, if the statements of the lemma hold for $(C, Q)$, they hold as well for some pointed curve over $\mathbb{C}$.

Now, any finite set of points of $C$ is defined over a finite field extension of $\mathbb{C}[[t]][1 / t]$. Replacing $S$ by its normalization in this field extension, we may assume that these are rational points of $X(\eta)$, and thus that there are sections of $f$ intersecting $X(\eta)$ at them. By making a further base extension, if necessary, and a sequence of blowups at the singular points of the special fiber, we may assume that the total space $X$ is regular, and that these sections factor through the smooth locus of $f$. The compensation for this is a change of the special fiber. However, the special fiber will have the same specification 
as the $C_{0}$ we described above. Thus, no confusion will ensue if we keep calling by $C_{0}$ this new fiber. Also, the section $\lambda$ can be extended to a section of this new family.

Now, let $P$ and $R$ be points of $C$ with $P$ distinct from $Q$ and $R$ distinct from $P$ and $Q$. As we mentioned above, we may assume there are sections $\gamma: S \rightarrow X$ and $\rho: S \rightarrow X$ through the smooth locus of $f$ such that $\gamma(\eta)=P$ and $\rho(\eta)=R$. Set $P_{0}:=\gamma(0)$ and $R_{0}:=\rho(0)$. Let $\Gamma$ and $\Lambda$ be the images of $\gamma$ and $\lambda$, respectively.

Fix nonnegative integers $i \leq i_{0}$ and $j \leq j_{0}$. Let $\omega$ be the relative dualizing bundle of $f: X \rightarrow S$. Let $V_{\eta}$ be the linear system of sections of the line bundle $\omega(\eta)((i+1) P+(j+1) Q)$ given by

$$
V_{\eta}:=H^{0}(\omega(\eta)((i+1) P))+H^{0}(\omega(\eta)((j+1) Q)) .
$$

Assume that $R$ is a ramification point of $V_{\eta}$. To prove the statements of the lemma hold for $(C, Q)$, it is enough to prove the following three statements:

1. For $i=0$, the system $V_{\eta}$ ramifies at $Q$ with weight $g$, and $R$ is a simple ramification point of $V_{\eta}$.

2. For $j=0$, the point $Q$ is a ramification point of $V_{\eta}$ of weight $g+i$ or $g+i+1$.

3. $\operatorname{dim} V_{\eta}(-(g+i+j) R) \leq 1$ and $V_{\eta}(-(g+i+j+2) R)=0$.

We will employ techniques of limit linear series, from [7], to show the above three statements. There are two cases to consider:

Case 1: Assume that $P_{0} \in E_{u}$ for some $u$.

Since $C_{0}$ is of compact type, there is an effective divisor $D$ of $X$ supported on $C_{0}$ such that, letting

$$
\mathcal{L}:=\omega((i+1) \Gamma+(j+1) \Lambda+D),
$$

we have $\left.\mathcal{L}\right|_{E_{m}} \cong \mathcal{O}_{E_{m}}$ for each $m=1, \ldots, n-1$,

$$
\left.\mathcal{L}\right|_{Z} \cong \omega_{Z}\left(\left(g_{Y}+i+j+3\right) B\right) \text { and }\left.\quad \mathcal{L}\right|_{Y} \cong \omega_{Y}\left(\left(1-g_{Y}\right) A\right) \text {. }
$$

Since, from the induction hypothesis, $A$ is a ramification point of weight $g_{Y}$ of the complete linear system of sections of $\omega_{Y}(A)$, the point $A$ is not a Weierstrass point of $Y$. Then $V:=H^{0}(X, \mathcal{L}) \cap V_{\eta}$ restricts to a linear system 
$V_{Z}$ of dimension $g+i+j$ of sections of $\omega_{Z}\left(\left(g_{Y}+i+j+3\right) B\right)$. Also from the induction hypothesis, $B$ is not a Weierstrass point of $Z$. So the order sequence of $B$ in the complete linear system of sections of $\omega_{Z}\left(\left(g_{Y}+i+j+3\right) B\right)$ is

$$
0,1, \ldots, g_{Y}+i+j+1, g_{Y}+i+j+3, \ldots, g+i+j+2 .
$$

As a consequence, the weight $w_{B}$ of $B$ as a ramification point of the linear system $V_{Z}$ satisfies

$$
w_{B} \leq 2\left(g_{Y}+i+j\right)+3 g_{Z},
$$

with equality if and only if $V_{Z}=H^{0}\left(\omega_{Z}\left(\left(g_{Y}+i+j+1\right) B\right)\right)$.

Analogously, choosing an appropriate $D$, we obtain a linear system $V_{Y}$ of dimension $g+i+j$ of sections of $\omega_{Y}\left(\left(g_{Z}+i+j+3\right) A\right)$, and the weight $w_{A}$ of $A$ as a ramification point of $V_{Y}$ satisfies

$$
w_{A} \leq 2\left(g_{Z}+i+j\right)+3 g_{Y},
$$

with equality if and only if $V_{Y}=H^{0}\left(\omega_{Y}\left(\left(g_{Z}+i+j+1\right) A\right)\right)$.

Let $r:=g+i+j-1$. Using the Plücker formula, the number $N$ of ramification points of $V_{Y}$ and $V_{Z}$ on $(Y-A) \cup(Z-B)$, counted with their respective weights, satisfies

$$
\begin{aligned}
N & =(r+1)\left(\left(2 g_{Z}+g_{Y}+i+j+1\right)+r\left(g_{Z}-1\right)\right)-w_{B} \\
& +(r+1)\left(\left(2 g_{Y}+g_{Z}+i+j+1\right)+r\left(g_{Y}-1\right)\right)-w_{A} \\
& =N^{\prime}+5 g+4(i+j)-w_{A}-w_{B},
\end{aligned}
$$

where

$$
N^{\prime}:=(r+1)((2 g+i+j)+r(g-1))-2 g-i-j .
$$

Now, from the theory of limit linear series, each one of the ramification points of $V_{Y}$ or $V_{Z}$ on $(Y-A) \cup(Z-B)$ is a limit of ramification points of $V_{\eta}$, and its weight as a ramification point is the sum of the weights of the ramification points of $V_{\eta}$ converging to it. Besides those, since $P$ and $Q$ are ramification points of $V_{\eta}$ with weights at least $g+j$ and $g+i$, respectively, the points $P_{0}$ and $Q_{0}$ appear as limits of ramification points of $V_{\eta}$ with weights summing up to at least $2 g+i+j$. Thus, from the Plücker formula, at most $N^{\prime}$ ramification points of $V_{\eta}$, counted with their weights, converge to $(Y-A) \cup(Z-B)$. So

$$
5 g+4(i+j)-w_{A}-w_{B} \leq 0 .
$$


However, Inequalities (5) and (6) for $w_{B}$ and $w_{A}$ yield the opposite inequality:

$$
5 g+4(i+j)-w_{A}-w_{B} \geq 0 .
$$

Thus, equalities hold, and hence

$$
V_{Y}=H^{0}\left(\omega_{Y}\left(\left(g_{Z}+i+j+1\right) A\right)\right) \quad \text { and } \quad V_{Z}=H^{0}\left(\omega_{Z}\left(\left(g_{Y}+i+j+1\right) B\right)\right) .
$$

In addition, $P$ and $Q$ are ramification points of $V_{\eta}$ of weights $g+j$ and $g+i$, respectively, and all the other ramification points of $V_{\eta}$ converge to $(Y-A) \cup(Z-B)$. In particular, Statement 2 and the first part of Statement 1 are shown.

Now, since $R$ is a ramification point of $V_{\eta}$, and $R$ is distinct from $P$ and $Q$, we have $R_{0} \in(Y-A) \cup(Z-B)$. So $R_{0}$ is a ramification point of either $V_{Y}$ or $V_{Z}$. From the induction hypothesis, the complete linear systems of sections of $\omega_{Y}\left(\left(g_{Z}+i+j+1\right) A\right)$ and $\omega_{Z}\left(\left(g_{Y}+i+j+1\right) B\right)$ have at most simple ramification points, other than $A$ or $B$. Thus $R$ is the unique ramification point of $V_{\eta}$ converging to $R_{0}$ and its weight is 1 . So the remainder of Statement 1 is shown.

As for Statement 3, assume, without loss of generality, that $R_{0} \in Z$. Set $n:=\operatorname{dim} V_{\eta}(-(g+i+j) R)$, and let $\sigma_{1}, \ldots, \sigma_{n}$ form a $\mathbb{C}[[t]]$-basis of $V \cap V_{\eta}(-(g+i+j) R)$. Their restrictions to $Z$ are sections of $V_{Z}$ vanishing with multiplicity at least $g+i+j$ on $R_{0}$. Assume, by contradiction, that $n \geq 2$. Since $R_{0}$ is a simple ramification point of $V_{Z}$, the sections $\left.\sigma_{1}\right|_{Z}, \ldots,\left.\sigma_{n}\right|_{Z}$ are linearly dependent. Thus, there is a nonzero $n$-tuple $\left(c_{1}, \ldots, c_{n}\right) \in \mathbb{C}^{n}$ such that $c_{1} \sigma_{1}+\cdots+c_{n} \sigma_{n}$ vanishes on $Z$, and hence on the whole $C_{0}$. Thus

$$
c_{1} \sigma_{1}+\cdots+c_{n} \sigma_{n}=t \sigma
$$

for some $\sigma \in H^{0}(X, \mathcal{L})$. Also $\sigma \in V_{\eta}(-(g+i+j) R)$, and hence $\sigma$ is a $\mathbb{C}[[t]]$ linear combination of $\sigma_{1}, \ldots, \sigma_{n}$. Plugging this linear combination in (7) we obtain a nontrivial $\mathbb{C}[[t]]$-linear relation among the sections $\sigma_{i}$, a contradiction. Thus $n \leq 1$. A similar analysis, using that $V_{Z}\left(-(g+i+j+2) R_{0}\right)=0$, shows that $V_{\eta}(-(g+i+2) R)=0$, finishing the proof of Statement 3 .

Case 2: Assume $P_{0}$ belongs to either $Y$ or $Z$.

Without loss of generality, we may assume that $P_{0} \in Z$. Again, since $C_{0}$ is of compact type, there is an effective divisor $D$ of $X$ supported on $C_{0}$ such that, letting

$$
\mathcal{L}:=\omega((i+1) \Gamma+(j+1) \Lambda+D),
$$


we have $\left.\mathcal{L}\right|_{E_{m}} \cong \mathcal{O}_{E_{m}}$ for each $m=1, \ldots, n-1$,

$$
\left.\mathcal{L}\right|_{Z} \cong \omega_{Z}\left((i+1) P_{0}+\left(g_{Y}+j+2\right) B\right) \quad \text { and }\left.\quad \mathcal{L}\right|_{Y} \cong \omega_{Y}\left(\left(1-g_{Y}\right) A\right)
$$

As before, $V:=H^{0}(X, \mathcal{L}) \cap V_{\eta}$ restricts to a linear system $V_{Z}$ of dimension $g+i+j$ of sections of $\omega_{Z}\left((i+1) P_{0}+\left(g_{Y}+j+2\right) B\right)$.

Now,

$$
V \supseteq H^{0}(X, \omega((i+1) \Gamma))+H^{0}(X, \omega((j+1) \Lambda+D)) .
$$

Reasoning as in Case 1 , we can show that $H^{0}(X, \omega((j+1) \Lambda+D))$ restricts to $H^{0}\left(\omega_{Z}\left(\left(g_{Y}+j+1\right) B\right)\right)$. On the other hand, the exact sequence

$$
0 \rightarrow H^{0}\left(\omega_{Z}\left((i+1) P_{0}\right)\right) \rightarrow H^{0}\left(\left.\omega((i+1) \Gamma)\right|_{C_{0}}\right) \rightarrow H^{0}\left(\omega_{Y}(A)\right)
$$

shows that $h^{0}\left(\left.\omega((i+1) \Gamma)\right|_{C_{0}}\right)=g+i$, and hence that $H^{0}(X, \omega((i+1) \Gamma))$ restricts to a vector subspace of $H^{0}\left(\omega_{Z}\left((i+1) P_{0}+B\right)\right)$ containing the subspace $H^{0}\left(\omega_{Z}\left((i+1) P_{0}\right)\right)$. Thus

$$
V_{Z} \supseteq H^{0}\left(\omega_{Z}\left((i+1) P_{0}\right)\right)+H^{0}\left(\omega_{Z}\left(\left(g_{Y}+j+1\right) B\right)\right),
$$

and a dimension count shows that equality holds.

The weight $w_{B}$ of $B$ as a ramification point of $V_{Z}$ depends on its weight as a ramification point of $V_{Z}\left(i, P_{0}\right)$. Now, from the induction hypothesis, $B$ is either an ordinary point or a simple ramification point of $V_{Z}\left(i, P_{0}\right)$. Hence, the order sequence at $B$ of the linear system $V_{Z}$ is either

$$
1,2, \ldots, g_{Y}+j, g_{Y}+j+2, g_{Y}+j+3, \ldots, g+i+j, g+i+j+1
$$

or

$$
1,2, \ldots, g_{Y}+j, g_{Y}+j+2, g_{Y}+j+3, \ldots, g+i+j, g+i+j+2 .
$$

At any rate,

$$
w_{B} \leq g_{Y}+j+2\left(g_{Z}+i\right)+1 \text {. }
$$

Notice that, if $i=0$, then $B$ is an ordinary point of $V_{Z}\left(0, P_{0}\right)$, as it is an ordinary point of $Z$, and thus Inequality (8) is strict.

On the other hand, let $D^{\prime}$ be an effective divisor of $X$ supported on $C_{0}$ such that, letting

$$
\mathcal{M}:=\omega\left((i+1) \Gamma+(j+1) \Lambda+D^{\prime}\right),
$$


we have $\left.\mathcal{M}\right|_{E_{m}} \cong \mathcal{O}_{E_{m}}$ for each $m=1, \ldots, n-1$,

$$
\left.\mathcal{M}\right|_{Y} \cong \omega_{Y}\left(\left(g_{Z}+i+j+3\right) A\right) \text { and }\left.\quad \mathcal{M}\right|_{Z} \cong \omega_{Z}\left((i+1) P_{0}-\left(g_{Z}+i\right) B\right) \text {. }
$$

Since, as mentioned above, $B$ is either an ordinary point or a simple ramification point of $V_{Z}\left(i, P_{0}\right)$, we have that $H^{0}(X, \mathcal{M}) \cap V_{\eta}$ restricts to a linear system $V_{Y}$ of dimension $g+i+j$ of sections of $\omega_{Y}\left(\left(g_{Z}+i+j+3\right) A\right)$.

Since $A$ is not a Weierstrass point of $Y$, the sequence of orders at $A$ of the complete linear system of sections of $\omega_{Y}\left(\left(g_{Z}+i+j+3\right) A\right)$ is

$$
0,1, \ldots, g_{Z}+i+j+1, g_{Z}+i+j+3, g_{Z}+i+j+4, \ldots, g+i+j+2 \text {. }
$$

Since $V_{Y}$ has codimension 2 in $H^{0}\left(\omega_{Y}\left(\left(g_{Z}+i+j+3\right) A\right)\right)$, the weight $w_{A}$ of $V_{Y}$ at $A$ satisfies

$$
w_{A} \leq 2\left(g_{Z}+i+j\right)+3 g_{Y}
$$

with equality if and only if $V_{Y}=H^{0}\left(\omega_{Y}\left(\left(g_{Z}+i+j+1\right) A\right)\right)$.

As in Case 1, using the Plücker formula, the number $N$ of ramification points of $V_{Y}$ and $V_{Z}$ on $(Y-A) \cup(Z-B)$, counted with their respective weights, satisfies

$$
N=N^{\prime}+4 g+4 i+3 j-w_{A}-w_{B}
$$

where

$$
N^{\prime}:=(g+i+j)(2 g+i+j)+(g+i+j)(g+i+j-1)(g-1)-g-i .
$$

As in Case 1 , since $Q$ is a ramification point of $V_{\eta}$ with weight at least $g+i$, there are at most $N^{\prime}$ ramification points of $V_{\eta}$, counted with their respective weights, converging to $(Y-A) \cup(Z-B)$. So

$$
4 g+4 i+3 j-w_{A}-w_{B} \leq 0 .
$$

On the other hand, Inequalities (8) and (9) yield

$$
w_{A}+w_{B} \leq 4 g+4 i+3 j+1 \text {. }
$$

In particular, $w_{A} \geq 2\left(g_{Z}+i+j\right)+3 g_{Y}-1$, whence

$$
V_{Y} \subset H^{0}\left(\omega_{Y}\left(\left(g_{Z}+i+j+2\right) A\right)\right) .
$$


Also, $Q$ has weight $g+i$ or $g+i+1$ in $V_{\eta}$. Thus Statement 2 is shown. Furthermore, if $i=0$ we have $w_{A}+w_{B}=4 g+4 i+3 j$. In this case, $V_{Y}=H^{0}\left(\omega_{Y}\left(\left(g_{Z}+i+j+1\right) A\right)\right)$ and $Q$ has weight $g+i$ in $V_{\eta}$, showing the first part of Statement 1 .

If $Q$ has weight $g+i+1$ in $V_{\eta}$, all other ramification points converge to $(Y-A) \cup(Z-B)$. If $Q$ has weight $g+i$, there is at most one ramification point of $V_{\eta}$, other than $Q$, converging outside $(Y-A) \cup(Z-B)$, and that point is simple. If $R$ is that point, then $\operatorname{dim} V_{\eta}(-(g+i+j) R) \leq 1$ and $V_{\eta}(-(g+i+j+2) R)=0$ because of the simplicity of $R$.

Assume now that $R_{0} \in(Y-A) \cup(Z-B)$. Let us first consider the case $R_{0} \in Y-A$. In this case, since, from the induction hypothesis, the complete linear system of sections of $\omega_{Y}\left(\left(g_{Z}+i+j+2\right) A\right)$ has at most simple ramification points, other than $A$, we have

$$
\begin{aligned}
h^{0}\left(\omega_{Y}\left(\left(g_{Z}+i+j+2\right) A-(g+i+j) R_{0}\right)\right. & \leq 1, \\
h^{0}\left(\omega_{Y}\left(\left(g_{Z}+i+j+2\right) A-(g+i+j+2) R_{0}\right)\right. & =0 .
\end{aligned}
$$

Thus $\operatorname{dim} V_{Y}\left(-(g+i+j) R_{0}\right) \leq 1$ and $V_{Y}\left(-(g+i+j+2) R_{0}\right)=0$ as well. It follows, as in Case 1, that

$$
\operatorname{dim} V_{\eta}(-(g+i+j) R) \leq 1 \quad \text { and } \quad V_{\eta}(-(g+i+j+2) R)=0 .
$$

Furthermore, if $i=0$, since in this case $V_{Y}=H^{0}\left(\omega_{Y}\left(\left(g_{Z}+i+j+1\right) A\right)\right)$, all the ramification points of $V_{Y}$ distinct from $A$ are simple. Thus $R_{0}$ is simple in $V_{Y}$, and hence $R$ is simple in $V_{\eta}$.

Assume now that $R_{0} \in Z-B$. There are two cases to consider. First, assume $R_{0}=P_{0}$. Since, by induction hypothesis, the complete linear system of sections of $\omega_{Z}\left(\left(g_{Y}+j+1\right) B\right)$ has at most simple ramification points other than $B$, the weight of $P_{0}$ as a ramification point of $V_{Z}$ is either $g+j$ or $g+j+1$. Since $P$ has at least weight $g+j$ in $V_{\eta}$, and $R \neq P$, the latter must hold, and $R$ must be a simple ramification point of $V_{\eta}$. In particular, $\operatorname{dim} V_{\eta}(-(g+i+j) R) \leq 1$ and $V_{\eta}(-(g+i+j+2) R)=0$.

Finally, assume $R_{0} \neq P_{0}$. Then

$$
\operatorname{dim} V_{Z}\left(-(g+i+j) R_{0}\right) \leq 1 \quad \text { and } \quad V_{Z}\left(-(g+i+j+2) R_{0}\right)=0
$$

from the induction hypothesis, and hence $\operatorname{dim} V_{\eta}(-(g+i+j) R) \leq 1$ and $V_{\eta}(-(g+i+j+2) R)=0$. Thus Statement 3 is shown. Also, if $i=0$, then $V_{Z}=H^{0}\left(\omega_{Z}\left(\left(g_{Y}+j+1\right) B\right)\right)$, and, since $R_{0} \neq P_{0}$, the weight of $R_{0}$ 
in $V_{Z}$ is equal to its weight in the complete linear system of sections of $\omega_{Z}\left(\left(g_{Y}+j+1\right) B\right)$. By induction hypothesis, this weight is one, and thus $R$ is a simple ramification point of $V_{\eta}$. So Statement 1 is shown.

3.3 Corollary. If $C$ is a general smooth curve of genus $g \geq 1$, then all its Weierstrass points are simple.

Proof. Apply Statement 1 of Proposition 3.1 for $i_{0}:=0$ and $i:=0$.

3.4 Proposition. Fix an integer $i_{0} \geq 0$. Let $C$ be a general smooth curve of genus $g \geq 1$. Then for any two distinct points $P$ and $R$ of $C$, and any nonnegative integer $i \leq i_{0}$,

$$
h^{0}\left(C, \omega_{C}((i+1) P-(g+i-2) R)\right)=2 .
$$

Proof. A line bundle of degree 2 on an elliptic curve has (at most) 2 linearly independent sections. Thus we may assume $g \geq 2$. Also, for $i=0$,

$$
h^{0}\left(C, \omega_{C}((i+1) P-(g+i-2) R)=h^{0}\left(C, \omega_{C}(-(g-2) R)\right)=2,\right.
$$

since $R$ is at most a simple Weierstrass point of $C$, a consequence of Corollary 3.3. So we need only show the stated equality for integers $i>0$.

For each integer $j \geq 2$ (resp. $j \geq 1$ ), let $M_{j}$ be the moduli space of smooth curves (resp. let $M_{j, 1}$ be the moduli space of smooth pointed curves) of genus $j$. Let $\bar{M}_{j}$ and $\bar{M}_{j, 1}$ denote their respective compactifications by stable (resp. stable, pointed) curves. For each positive integer $i \leq i_{0}$, let $D^{(i)} \subseteq M_{g+i}$ be the subset parameterizing curves admitting a covering of degree at most $g+i-2$ of the projective line totally ramified at a point. By [1], Thm. 3.11, p. 333, the subvariety $D^{(i)}$ is irreducible of codimension 2. Let $\bar{D}^{(i)} \subset \bar{M}_{g+i}$ be the closure of $D^{(i)}$.

Let $\mu_{i}: M_{g, 1} \times M_{i, 1} \rightarrow \bar{M}_{g+i}$ be the natural map, associating to a pair of smooth pointed curves the stable uninodal curve which is the union of these curves identified at the marked points. Let $E^{(i)}:=\mu_{i}^{-1}\left(\bar{D}^{(i)}\right)$. Let $\rho_{i}: E^{(i)} \rightarrow M_{g}$ be the natural map, forgetting the second pointed curve and the marked point on the first curve. Since $C$ is general, we may assume that, for each $i=1, \ldots, i_{0}$, the curve $C$ is parameterized by a point of $M_{g}$ over which the fiber of $\rho_{i}$ has minimum dimension. We claim this dimension is at most $3 i-3$, whence less than $\operatorname{dim} M_{i, 1}$. Indeed, if the dimension were larger, then $E^{(i)}$ would have codimension at most 1 in $M_{g, 1} \times M_{i, 1}$, and 
hence would dominate $\bar{D}^{(i)}$ under $\mu_{i}$. So $\bar{D}^{(i)}$ would be contained in the boundary $\bar{M}_{g+i}-M_{g+i}$, an absurd. From the claim, for each $i=1, \ldots, i_{0}$, the general smooth pointed curve $\left(Y_{i}, B_{i}\right)$ of genus $i$ is such that, for any $P \in C$, the pair of pointed curves $\left((C, P),\left(Y_{i}, B_{i}\right)\right)$ is not parameterized by $E^{(i)}$. Consequently, the stable uninodal curve $X_{i}$, union of $C$ and $Y_{i}$ with $P$ and $B_{i}$ identified, is parameterized by a point of $\bar{M}_{g+i}$ off $\bar{D}^{(i)}$, for each $i=1, \ldots, i_{0}$.

Suppose, by contradiction, that for certain distinct points $P$ and $Q$ of $C$, and a certain positive integer $i \leq i_{0}$, we have

$$
h^{0}\left(C, \omega_{C}((i+1) P-(g+i-2) Q) \geq 3 .\right.
$$

Put $g^{\prime}:=g+i$. Since, by Riemann-Roch, $h^{0}\left(C, \omega_{C}((i+1) P-i Q)\right)=g$, there is an integer $j$ with $2 \leq j<g$ such that

$h^{0}\left(C, \omega_{C}\left((i+1) P-\left(g^{\prime}-j\right) Q\right)=h^{0}\left(C, \omega_{C}\left((i+1) P-\left(g^{\prime}-j-1\right) Q\right)=j+1\right.\right.$.

Again by Riemann-Roch,

$$
h^{0}\left(C, \mathcal{O}_{C}\left(\left(g^{\prime}-j\right) Q-(i+1) P\right)\right)>h^{0}\left(C, \mathcal{O}_{C}\left(\left(g^{\prime}-j-1\right) Q-(i+1) P\right)\right) .
$$

Thus, there is a map $\phi: C \longrightarrow \mathbb{P}^{1}$ of degree $g^{\prime}-j$ such that $\phi^{*}(0)=\left(g^{\prime}-j\right) Q$ and $\phi^{*}(\infty) \geq(i+1) P$. Let $i^{\prime}$ be the integer such that $i^{\prime}+1$ is the multiplicity of $P$ in $\phi^{*}(\infty)$. Then $i^{\prime} \geq i$.

Set $Y:=Y_{i}$ and $B:=B_{i}$. Since $B$ is general, $B$ is not a Weierstrass point of $Y$. Thus, since $i^{\prime} \geq i$, we have $h^{0}\left(Y, \mathcal{O}_{Y}\left(i^{\prime} B\right)\right)<h^{0}\left(Y, \mathcal{O}_{Y}\left(\left(i^{\prime}+1\right) B\right)\right)$. So, there is a map $\psi: Y \longrightarrow \mathbb{P}^{1}$ of degree $i^{\prime}+1$ such that $\psi^{*}(\infty)=\left(i^{\prime}+1\right) B$.

Putting together the maps $\phi$ and $\psi$, we may construct the covering with source $X_{i}$ depicted in Figure 1 below, which can be represented by a point $\left[X_{i}\right]$ of the (compactification of the) Hurwitz scheme parameterizing (pseudo)admissible coverings of the projective line of degree $\left(g^{\prime}-j\right)$ totally ramified at a point; see Remark 3.5. Since coverings of $\mathbb{P}^{1}$ form a dense open subscheme of this compactification, the curve $X_{i}$ is limit of smooth curves equipped with a degree- $\left(g^{\prime}-j\right)$ map to the projective line totally ramified at a point. Since $j \geq 2$, it follows that $\left[X_{i}\right]$ lies on the boundary of $D^{(i)}$, a contradiction.

3.5 Remark. The Hurwitz scheme we used in the proof of Proposition 3.4 is mentioned in [6], Section 5. It can be constructed following the same 


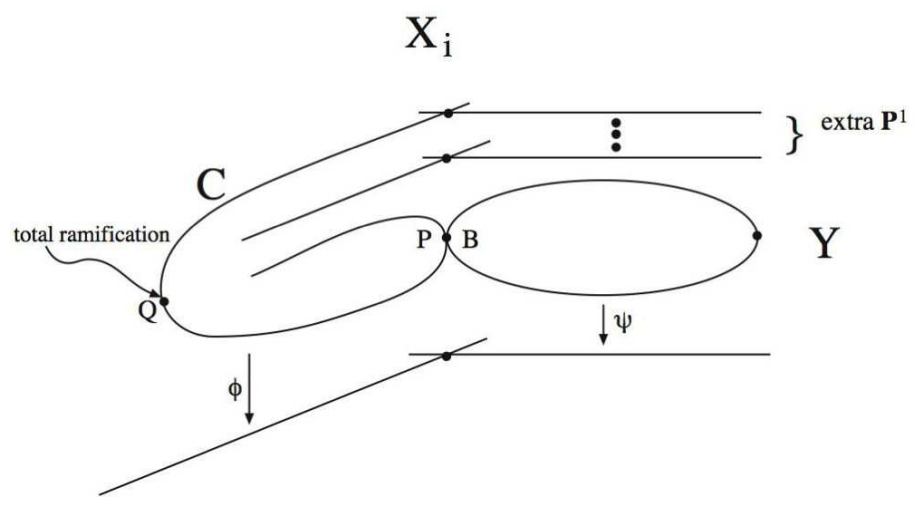

Figure 1: The covering.

reasoning used in the construction of the Hurwitz scheme of (simple) admissible coverings, given in the proof of [10], Thm. 4, p. 58. Also, the local descriptions of both schemes are the same, given on [10], p. 62. From this description we see that the Hurwitz scheme is equidimensional. Now, there is a natural forgetful map from the Hurwitz scheme to a corresponding moduli space of pointed genus- 0 curves, taking a covering to its target. This map is finite and surjective, also by [10, Thm. 4, p. 58. Since the moduli spaces of pointed genus-0 curves are irreducible (see [12] or [11]), it follows that each irreducible component of the Hurwitz scheme covers the target. So coverings of $\mathbb{P}^{1}$ form a dense open subscheme of the Hurwitz scheme, a fact used in the proof of Proposition 3.4 .

3.6 Remark. We tried to prove Proposition 3.4 using the same induction argument used in the proof of Lemma 3.2. However, we could not prove the initial step, that is, the following statement: Let $C$ be a general elliptic curve, $Q \in C$ a general point, and $P \in C-\{Q\}$ any point. Let $i$ and $j$ be nonnegative integers. Then the linear system $V$ of sections of the line bundle $\omega_{C}((i+1) P+(j+1) Q)$ generated by $H^{0}\left(\omega_{C}((i+1) P)\right)$ and $H^{0}\left(\omega_{C}((j+1) Q)\right)$ satisfies $\operatorname{dim} V(-(i+j-1) R)=2$ for each $R \in C-\{P, Q\}$. 


\section{Weierstrass divisors}

4.1 (Wronski maps) Let $C$ be a smooth curve of genus $g$. For each integer $j \geq 0$, consider the family of linear systems $V_{C}(j, P)$ for $P$ varying on $C$. More precisely, let $p_{1}$ and $p_{2}$ denote the projections of $C \times C$ onto the first and second factors, and $\Delta \subset C \times C$ the diagonal. The relative canonical bundle of $p_{1}$ is simply the pullback $p_{2}^{*} \omega_{C}$ of the canonical bundle $\omega_{C}$ of $C$. For each integer $j \geq 0$, let

$$
\mathcal{L}_{j}:=p_{2}^{*} \omega_{C}((j+1) \Delta), \quad \mathcal{E}_{j}:=p_{1 *} \mathcal{L}_{j} .
$$

Notice that, for each point $P$ of $C$, identifying $\{P\} \times C$ with $C$ in the natural way, $\left.\mathcal{L}_{j}\right|_{\{P\} \times C}=\omega_{C}((j+1) P)$. Also, as $h^{0}\left(\omega_{C}((j+1) P)\right)=g+j$ for every $P \in C$, the sheaf $\mathcal{E}_{j}$ is a bundle of rank $g+j$ and $\left.\mathcal{E}_{j}\right|_{P}=H^{0}\left(\omega_{C}((j+1) P)\right)$.

For each integer $\ell \geq 0$ and each line bundle $\mathcal{M}$ on $C \times C$, let $\mathcal{J}_{p_{1}}^{\ell}(\mathcal{M})$ be the bundle of rank $\ell+1$ of $p_{1}$-relative jets of $\mathcal{M}$ truncated in order $\ell$. Let

$$
\rho_{j, \ell}: p_{1}^{*} \mathcal{E}_{j} \rightarrow \mathcal{J}_{p_{1}}^{\ell}\left(\mathcal{L}_{j}\right)
$$

be the map of bundles locally obtained by differentiating up to order $\ell$ along the fibers of $p_{1}$ the sections of $\mathcal{L}_{j}$. We call $\rho_{j, \ell}$ a Wronski map.

The map $\rho_{j, g+j-1}$ is a map of bundles of the same rank. Taking determinants, we get a section $z_{j}$ of the line bundle

$$
\bigwedge^{g+j} \mathcal{J}_{p_{1}}^{g+j-1}\left(\mathcal{L}_{j}\right) \otimes \bigwedge^{g+j} p_{1}^{*} \mathcal{E}_{j}^{\vee}
$$

which is naturally isomorphic, using the truncation sequence of the bundles of jets, to

$$
p_{2}^{*} \omega_{C}((j+1) \Delta)^{\otimes g+j} \otimes p_{2}^{*} \omega_{C}^{\otimes(g+j)(g+j-1) / 2} \otimes \bigwedge^{g+j} p_{1}^{*} \mathcal{E}_{j}^{\vee}
$$

or more simply to

$$
p_{2}^{*} \omega_{C}^{\otimes(g+j)(g+j+1) / 2}((g+j)(j+1) \Delta) \otimes \bigwedge^{g+j} p_{1}^{*} \mathcal{E}_{j}^{\vee}
$$

4.2 (Weierstrass divisors.) Keep the notation used in Subsection 4.1. Let $Z_{j} \subseteq C \times C$ denote the zero scheme of $z_{j}$. The section $z_{j}$ is a relative wronskian. More precisely, for each $P \in C$, on $\{P\} \times C$, identified with $C$ in 
the natural way, the section $z_{j}$ restricts to the wronskian of the linear system $V_{C}(j, P)$. Hence, $Z_{j}$ consists of the pairs $(P, Q) \in C \times C$ such that $V_{C}(j, P)$ ramifies at $Q$. Now, since $z_{j}$ is nonzero, being so on each fiber, $Z_{j}$ is a Cartier divisor. By Proposition 2.2 , the divisor $Z_{j}$ intersects each fiber $\{P\} \times C$ at $(P, P)$ with multiplicity $g+\operatorname{wt}(P)$, where wt $(P)$ is the weight of $P$ in the canonical system of $C$. Thus $Z_{j}$ contains $\Delta$ with multiplicity exactly $g$. Let

$$
W_{j}:=Z_{j}-g \Delta .
$$

Then $W_{j}$ is, set-theoretically, the locus of pairs $(P, Q) \in C \times C$ such that either $P=Q$ and $P$ is a Weierstrass point of $C$, or $P \neq Q$ and $Q$ is a ramification point of $V_{C}(j, P)$. We call $W_{j}$ the $j$-th Weierstrass divisor of $C$.

4.3 Proposition. Let $C$ be a smooth curve of genus $g \geq 1$ and $j$ a nonnegative integer. Let $\Delta$ be the diagonal of $C \times C$, and $p_{1}$ and $p_{2}$ the projections of $C \times C$ onto the indicated factors. Let $\omega_{C}$ be the canonical bundle of $C$, and set $K_{\ell}:=c_{1}\left(p_{\ell}^{*} \omega_{C}\right)$ for $\ell=1,2$. Let $W_{j} \subseteq C \times C$ be the $j$-th Weierstrass divisor of $C$. Then its class $\left[W_{j}\right]$ in the Chow group of $C \times C$ satisfies

$$
\left[W_{j}\right]=\frac{1}{2}(g+j)(g+j+1) K_{2}+j(g+j+1)[\Delta]+\frac{1}{2} j(j+1) K_{1} .
$$

Proof. Use the notation in Subsections 4.1 and 4.2. Since $W_{j}=Z_{j}-g \Delta$, and $Z_{j}$ is the zero scheme of a section of the line bundle

$$
p_{2}^{*} \omega_{C}^{\otimes(g+j)(g+j+1) / 2}((g+j)(j+1) \Delta) \otimes \bigwedge^{g+j} p_{1}^{*} \mathcal{E}_{j}^{\vee}
$$

we get

$$
\left[W_{j}\right]=\frac{1}{2}(g+j)(g+j+1) K_{2}+j(g+j+1)[\Delta]-p_{1}^{*} c_{1}\left(\mathcal{E}_{j}\right) .
$$

To finish, we need only show that

$$
c_{1}\left(\mathcal{E}_{j}\right)=-\frac{1}{2} j(j+1) c_{1}\left(\omega_{C}\right) .
$$

We show (13) by induction on $j$. First of all,

$$
\mathcal{E}_{0}=p_{1 *} p_{2}^{*} \omega_{C}=H^{0}\left(\omega_{C}\right) \otimes \mathcal{O}_{C} .
$$

Since $\mathcal{E}_{0}$ is free, $c_{1}\left(\mathcal{E}_{0}\right)=0$. 
Assume now that $j>0$ and $c_{1}\left(\mathcal{E}_{j-1}\right)=-(j(j-1) / 2) c_{1}\left(\omega_{C}\right)$. Consider the natural short exact sequence

$$
\left.0 \rightarrow p_{2}^{*} \omega_{C}(j \Delta) \rightarrow p_{2}^{*} \omega_{C}((j+1) \Delta) \rightarrow p_{2}^{*} \omega_{C}((j+1) \Delta)\right|_{\Delta} \rightarrow 0 .
$$

Since $H^{1}\left(\omega_{C}(j P)\right)=0$ for each $P \in C$, applying $p_{1 *}$ to the sequence above, we get the exact sequence

$$
\left.0 \rightarrow \mathcal{E}_{j-1} \rightarrow \mathcal{E}_{j} \rightarrow p_{1 *} p_{2}^{*} \omega_{C}((j+1) \Delta)\right|_{\Delta} \rightarrow 0 .
$$

Now, $\left.p_{\ell}\right|_{\Delta}$ is an isomorphism for $\ell=1,2$. So $\left.p_{1 *} p_{2}^{*} \omega_{C}\right|_{\Delta}=\omega_{C}$. In addition, $\left.p_{1 *} \mathcal{O}_{C \times C}(-\Delta)\right|_{\Delta}=\omega_{C}$. Thus

$$
\begin{aligned}
c_{1}\left(\mathcal{E}_{j}\right) & =c_{1}\left(\mathcal{E}_{j-1}\right)+c_{1}\left(\left.p_{1 *} p_{2}^{*} \omega_{C}((j+1) \Delta)\right|_{\Delta}\right) \\
& =-(j(j-1) / 2) c_{1}\left(\omega_{C}\right)+(1-(j+1)) c_{1}\left(\omega_{C}\right) \\
& =-(j(j+1) / 2) c_{1}\left(\omega_{C}\right),
\end{aligned}
$$

as claimed.

4.4 Proposition. Let $C$ be a general smooth curve of genus $g \geq 1$ and $j$ a nonnegative integer. Let $W_{j} \subseteq C \times C$ be the $j$-th Weierstrass divisor of $C$. Then $W_{j}$ is nonsingular and intersects the diagonal $\Delta$ transversally, at the pairs $(P, P)$ such that $P$ is a Weierstrass point of $C$.

Proof. Let us show first that $W_{j}$ intersects $\Delta$ transversally. As pointed out in Subsection 4.2, the intersection $W_{j} \cap \Delta$ is, set-theoretically, the set of pairs $(P, P)$ such that $P$ is a Weierstrass point of $C$. As $C$ is general, by Corollary 3.3, all its Weierstrass points are simple, and number $g^{3}-g$ by Plücker Formula. Now, since the intersection $W_{j} \cap \Delta$ is finite, the number of points of intersection, weighted by their intersection multiplicities, is equal to the degree of the product $\left[W_{j}\right][\Delta]$. Using the notation and Formula (11) of Proposition 4.3, and using the Formulas

$$
\int_{C \times C} K_{2}[\Delta]=\int_{C \times C} K_{1}[\Delta]=-\int_{C \times C}[\Delta]^{2}=2 g-2
$$

and

$$
\int_{C \times C} K_{1} K_{2}=4(g-1)^{2},
$$

we get

$\int_{C \times C}\left[W_{j}\right][\Delta]=(g+j)(g+j+1)(g-1)-2 j(g+j+1)(g-1)+j(j+1)(g-1)$, 
which is exactly $g^{3}-g$. Thus the intersection multiplicities are all one.

As a corollary of the transversal intersection, $W_{j}$ is nonsingular at its points on $\Delta$. So, let now $(P, Q) \in W_{j}$ for $P$ and $Q$ distinct, and let us show that $W_{j}$ is nonsingular at $(P, Q)$ as well.

Let $J:=\operatorname{Pic}^{g-1}(C)$, the component of the Picard scheme of $C$ parameterizing line bundles of degree $g-1$. Let $\Theta \subset J$ be the theta divisor, parameterizing line bundles with nontrivial global sections. Let

$$
\mu: C \times C \rightarrow J
$$

be the map taking a pair $(R, S)$ to the point of $J$ representing the bundle $\omega_{C}((j+1) R-(g+j) S)$.

We claim that $\mu\left(W_{j}\right) \subseteq \Theta$. Indeed, let $C^{(3)}:=C \times C \times C$, and denote by $p_{1,2}$ and $p_{3}$ the projection maps of $C^{(3)}$ onto the indicated factors. Let $\Delta_{1,3}$ and $\Delta_{2,3}$ be the indicated diagonals of $C^{(3)}$. Set

$$
\mathcal{F}:=p_{3}^{*} \omega_{C}\left((j+1) \Delta_{1,3}-(g+j) \Delta_{2,3}\right) .
$$

Recall the notation of Subsection 4.1. From the construction of $\Theta$, to show that $\mu\left(W_{j}\right) \subseteq \Theta$, it is enough to show that the Wronski map $\rho_{j, g+j-1}$ represents universally the cohomology of $\mathcal{F}$ or, put more simply, that $\rho_{j, g+j-1}$ can be viewed as a presentation of the right derived image $R^{1}\left(p_{1,2}\right)_{*} \mathcal{F}$.

Let $\mathcal{G}:=p_{3}^{*} \omega_{C}\left((j+1) \Delta_{1,3}\right)$. Then $\mathcal{F} \subseteq \mathcal{G}$. From the definition of the Wronski map $\rho_{j, g+j-1}$, we get that $\rho_{j, g+j-1}$ is the image under $\left(p_{1,2}\right)_{*}$ of the quotient map $\mathcal{G} \rightarrow \mathcal{G} / \mathcal{F}$. Thus, the map $\rho_{j, g+j-1}$ is the first map in the following piece of the long derived sequence of $0 \rightarrow \mathcal{F} \rightarrow \mathcal{G} \rightarrow \mathcal{G} / \mathcal{F} \rightarrow 0$ under $\left(p_{1,2}\right)_{*}$ :

$$
\left(p_{1,2}\right)_{*} \mathcal{G} \rightarrow\left(p_{1,2}\right)_{*}(\mathcal{G} / \mathcal{F}) \rightarrow R^{1}\left(p_{1,2}\right)_{*} \mathcal{F} \rightarrow R^{1}\left(p_{1,2}\right)_{*} \mathcal{G}
$$

Now, a fiberwise analysis shows that $R^{1}\left(p_{1,2}\right)_{*} \mathcal{G}=0$. Thus $\rho_{j, g+j-1}$ is a presentation for $R^{1}\left(p_{1,2}\right)_{*} \mathcal{F}$, finishing the proof that $\mu\left(W_{j}\right) \subseteq \Theta$.

Let $\mathcal{L}:=\omega_{C}((j+1) P-(g+j) Q)$, and denote by $[\mathcal{L}]$ the point of $J$ representing $\mathcal{L}$. Since $(P, Q) \in W_{j}$, we have $[\mathcal{L}] \in \Theta$. By Proposition 3.1 , $h^{0}(C, \mathcal{L})=1$. Thus, it follows from [2], Prop. $(4.2)$, p. 189, that $[\mathcal{L}]$ is a nonsingular point of $\Theta$. Furthermore, identifying the cotangent space of $J$ at $[\mathcal{L}]$ with $H^{0}\left(C, \omega_{C}\right)$, the cotangent space of $\Theta$ at $[\mathcal{L}]$ is the quotient by the subspace $H^{0}\left(C, \omega_{C}(-F)\right)$, where $F$ is the unique effective divisor of $C$ such that $\mathcal{L}=\mathcal{O}_{C}(F)$. 
Identifying the cotangent space of $C \times C$ at $(P, Q)$ with $\left.\left.\omega_{C}\right|_{P} \oplus \omega_{C}\right|_{Q}$, the induced map of cotangent spaces $d \mu^{*}: T_{J,[\mathcal{L}]}^{*} \rightarrow T_{C \times C,(P, Q)}^{*}$ is equivalent to the evaluation map,

$$
\epsilon:\left.\left.H^{0}\left(C, \omega_{C}\right) \rightarrow \omega_{C}\right|_{P} \oplus \omega_{C}\right|_{Q} .
$$

We claim that $\epsilon\left(H^{0}\left(C, \omega_{C}(-F)\right)\right) \neq 0$. Indeed, if that were not the case, we would have $H^{0}\left(C, \omega_{C}(-F)\right)=H^{0}\left(C, \omega_{C}(-F-P-Q)\right)$, that is,

$$
h^{0}\left(C, \mathcal{O}_{C}((g+j-1) Q-(j+2) P)\right)=h^{0}\left(C, \mathcal{O}_{C}((g+j) Q-(j+1) P)\right) .
$$

By the Riemann-Roch theorem,

$$
h^{0}\left(C, \mathcal{O}_{C}((g+j) Q-(j+1) P)\right)=h^{0}(C, \mathcal{L})=1,
$$

and thus, also by the Riemann-Roch theorem,

$$
h^{0}\left(C, \omega_{C}((j+2) P-(g+j-1) Q)\right)=3 .
$$

However, this contradicts Proposition 3.4 .

Since $\mu\left(W_{j}\right) \subseteq \Theta$, the image of $\epsilon\left(H^{0}\left(C, \omega_{C}(-F)\right)\right)$ in the cotangent space of $W_{j}$ at $(P, Q)$ is zero. Since $\epsilon\left(H^{0}\left(C, \omega_{C}(-F)\right)\right) \neq 0$, that cotangent space is a proper quotient of the cotangent space of $C \times C$ at $(P, Q)$, and thus has dimension at most 1 . Since $W_{j}$ is a divisor, it follows that $W_{j}$ is nonsingular at $(P, Q)$.

\section{Special ramification classes}

5.1 (Special ramification loci.) Let $C$ be a smooth curve of genus $g \geq 1$. For each nonnegative integer $i$, consider the following loci in $C \times C$ :

1. The locus $D_{i}^{+}$of pairs $(P, Q) \in C \times C$ such that

$$
(g+i-1) Q-(i+1) P
$$

is linearly equivalent to an effective divisor.

2. The locus $E_{i}^{+}$of pairs $(P, Q) \in C \times C$ such that

$$
(g+i+1) Q-(i+1) P
$$

is linearly equivalent to a moving effective divisor. 
3. The locus $S W_{i}^{+}$of pairs $(P, Q) \in C \times C$ such that $Q$ is a special ramification point of $V_{C}(i, P)$.

We claim that, set-theoretically,

$$
S W_{i}^{+}=D_{i}^{+} \cup E_{i}^{+} .
$$

Indeed, by the Riemann-Roch Theorem, for a pair $(P, Q) \in C \times C$, the divisor $(g+i-1) Q-(i+1) P$ is linearly equivalent to an effective one if and only if

$$
h^{0}\left(\omega_{C}((i+1) P-(g+i-1) Q)\right) \geq 2,
$$

while $(g+i+1) Q-(i+1) P$ is linearly equivalent to a moving effective divisor if and only if

$$
h^{0}\left(\omega_{C}((i+1) P-(g+i+1) Q)\right) \geq 1 .
$$

At any rate, if $(P, Q) \in D_{i}^{+} \cup E_{i}^{+}$, then $Q$ is a special ramification point of $V_{C}(i, P)$, that is, $(P, Q) \in S W_{i}^{+}$

On the other hand, let $(P, Q) \in C \times C-\left(D_{i}^{+} \cup E_{i}^{+}\right)$. Then

$$
\begin{aligned}
& h^{0}\left(\omega_{C}((i+1) P-(g+i-1) Q)\right)=1, \\
& h^{0}\left(\omega_{C}((i+1) P-(g+i+1) Q)\right)=0 .
\end{aligned}
$$

So, either $Q$ is an ordinary or a simple ramification point of $V_{C}(i, P)$, that is, $(P, Q) \notin S W_{i}^{+}$.

Let $\Delta$ be the diagonal subscheme of $C \times C$. Notice that $E_{i}^{+} \cap \Delta$ consists of the pairs $(P, P)$ such that $P$ is a Weierstrass point of $C$. However, if $g>1$, both $D_{i}^{+}$and $S W_{i}^{+}$contain $\Delta$. (If $g=1$, then $D_{i}^{+}=E_{i}^{+}=S W_{i}^{+}=\emptyset$.)

Let $D_{i}, E_{i}$ and $S W_{i}$ be the loci of points in $D_{i}^{+}, E_{i}^{+}$and $S W_{i}^{+}$that lie off $\Delta$. Of course, Expression (16) implies $S W_{i}=D_{i} \cup E_{i}$. Our Proposition 5.4 claims that, if $C$ is general, then $S W_{i}=D_{i} \cup E_{i}$ holds in a more refined way, in the cycle group of $C \times C$. Before stating it, we need to endow $D_{i}, E_{i}$ and $S W_{i}$ with natural subscheme structures.

5.2 (Special ramification schemes) Keep the notation of Subsection 5.1, and recall that of Subsections 4.1 and 4.2. Notice that the subsets $D_{i}^{+}$and $E_{i}^{+}$ are the supports of the degeneracy schemes of $\rho_{i, g+i-2}$ and $\rho_{i, g+i}$, respectively. So we may give $D_{i}^{+}$and $E_{i}^{+}$the corresponding scheme structures. Give $D_{i}$ and $E_{i}$ the corresponding open subscheme structures. We say that $D_{i}$ and $E_{i}$ are the $i$-th special ramification schemes of type Diaz and Cukierman, 
respectively. Call $E_{i}^{+}$the $i$-th expanded special ramification scheme of type Cukierman.

In addition, differentiating along the fibers of $p_{1}$ a section of $\mathcal{O}_{C \times C}\left(Z_{i}\right)$ defining $Z_{i}$, we obtain a section of $\mathcal{J}_{p_{1}}^{1}\left(\mathcal{O}_{C \times C}\left(Z_{i}\right)\right)$, well-defined modulo $\mathbb{C}^{*}$. By functoriality, its zero scheme contains a pair $(P, Q)$ if and only if $Q$ is a special Weierstrass point of $V_{C}(i, P)$. Thus the zero scheme gives a scheme structure for $S W_{i}^{+}$. Give $S W_{i}$ the induced open subscheme structure. We say that $S W_{i}$ is the $i$-th special ramification scheme of $C$.

Now, $Z_{i}=W_{i}+g \Delta$. As done for $Z_{i}$, we can differentiate along the fibers of $p_{1}$ a section of $\mathcal{O}_{C \times C}\left(W_{i}\right)$ defining $W_{i}$ to obtain a section of $\mathcal{J}_{p_{1}}^{1}\left(\mathcal{O}_{C \times C}\left(W_{i}\right)\right)$. Its zero scheme $S$ coincides with the scheme $S W_{i}$ off $\Delta$, because $Z_{i}$ coincides with $W_{i}$ there. Moreover, if $C$ is general, then $S$ does not intersect $\Delta$, and hence $S=S W_{i}$ scheme-theoretically. Indeed, let $P$ be a point of $C$. If $(P, P) \in W_{i}$, then $P$ is a Weierstrass point of $C$. Moreover, as $C$ is general, by Corollary 3.3 , the point $P$ is a simple Weierstrass point. So, it follows from Proposition 2.2 that $W_{i}$ intersects the fiber $\{P\} \times C$ transversally at $(P, P)$. Thus the derivative along $\{P\} \times C$ of a section defining $W_{i}$ does not vanish at $(P, P)$. So $S \cap \Delta=\emptyset$.

5.3 Lemma. Let $\mathcal{O}$ be a local ring, and $r$ a nonnegative integer. Let $M$ be a matrix with $r+2$ rows and $r+1$ columns and entries in $\mathcal{O}$. Let $M_{1}$ and $M_{2}$ be the submatrices obtained from $M$ by removing the last row, and the last two rows, respectively. Assume that the matrix obtained from $M_{1}$ by taking residues has rank at least $r$. Let $z$ denote the determinant of $M_{1}$. Then there are $u, v \in \mathcal{O}$ such that

1. $(z, u)$ is the ideal of all maximal minors of $M_{2}$,

2. $(z, v)$ is the ideal of all maximal minors of $M$,

3. $(z, u v)$ is the ideal generated by the two maximal minors of $M$ obtained by removing each of the last two rows.

Proof. We may write $M$ in the form

$$
M=\left[\begin{array}{lll}
A & a & b \\
c & f_{1} & f_{2} \\
d & g_{1} & g_{2} \\
e & h_{1} & h_{2}
\end{array}\right],
$$


where $A$ is a square matrix of size $r-1$, where $a$ and $b$ are column vectors of size $r-1$, where $c, d$ and $e$ are row vectors of size $r-1$, and where $f_{1}, f_{2}$, $g_{1}, g_{2}, h_{1}$ and $h_{2}$ are elements of $\mathcal{O}$.

Let $I$ and $J$ be the ideals of $\mathcal{O}$ generated, respectively, by all maximal minors of the submatrices

$$
M_{2}=\left[\begin{array}{ccc}
A & a & b \\
c & f_{1} & f_{2}
\end{array}\right] \text { and } M=\left[\begin{array}{ccc}
A & a & b \\
c & f_{1} & f_{2} \\
d & g_{1} & g_{2} \\
e & h_{1} & h_{2}
\end{array}\right] .
$$

Also, let $K \subseteq \mathcal{O}$ be the ideal generated by the determinants of the square submatrices

$$
M_{1}=\left[\begin{array}{ccc}
A & a & b \\
c & f_{1} & f_{2} \\
d & g_{1} & g_{2}
\end{array}\right] \text { and } M_{1}^{\prime}:=\left[\begin{array}{ccc}
A & a & b \\
c & f_{1} & f_{2} \\
e & h_{1} & h_{2}
\end{array}\right] .
$$

Notice that the determinant of the first matrix is $z$.

From the hypothesis, the matrix obtained from $M_{2}$ by taking residues has rank at least $r-1$. Thus, performing row and column operations on $M$, including column and row exchanges, we may, without changing the ideals $I$, $J$ and $K$, assume that $A$ is the identity matrix, $a=b=0$ and $c=d=e=0$. Then $z=f_{1} g_{2}-f_{2} g_{1}$ and

$$
\begin{aligned}
I & =\left(f_{1}, f_{2}\right), \\
J & =\left(f_{1} g_{2}-f_{2} g_{1}, f_{1} h_{2}-f_{2} h_{1}, g_{1} h_{2}-g_{2} h_{1}\right), \\
K & =\left(f_{1} g_{2}-f_{2} g_{1}, f_{1} h_{2}-f_{2} h_{1}\right) .
\end{aligned}
$$

Now, since the matrix obtained from $M_{1}$ by taking residues has rank at least $r$, at least one among $f_{1}, f_{2}, g_{1}, g_{2}$ is invertible.

If $f_{1}$ is invertible, then

$$
g_{1} h_{2}-g_{2} h_{1}=\left(g_{1} / f_{1}\right)\left(f_{1} h_{2}-f_{2} h_{1}\right)-\left(h_{1} / f_{1}\right)\left(f_{1} g_{2}-f_{2} g_{1}\right) .
$$

Thus, the lemma holds for $u=1$ and $v=f_{1} h_{2}-f_{2} h_{1}$. The case where $f_{2}$ is invertible is similar.

If $g_{1}$ is invertible, then

$$
\begin{aligned}
\left(f_{1} h_{2}-f_{2} h_{1}\right) & =\left(f_{1} / g_{1}\right)\left(g_{1} h_{2}-g_{2} h_{1}\right)+\left(h_{1} / g_{1}\right)\left(f_{1} g_{2}-f_{2} g_{1}\right), \\
f_{2} & =\left(g_{2} / g_{1}\right) f_{1}-\left(1 / g_{1}\right)\left(f_{1} g_{2}-f_{2} g_{1}\right) .
\end{aligned}
$$


Thus the lemma holds for $u=f_{1}$ and $v=g_{1} h_{2}-g_{2} h_{1}$. A similar analysis holds if $g_{2}$ is invertible.

5.4 Proposition. Let $C$ be a general smooth curve of genus $g \geq 1$ and $i$ a nonnegative integer. Let $\Delta$ be the diagonal of $C \times C$ and $W_{i}$ the $i$-th Weierstrass divisor. Let $S W_{i}$ be the $i$-th special ramification scheme, and $D_{i}$ and $E_{i}$ the $i$-th special ramification schemes of type Diaz and Cukierman, respectively. Let $E_{i}^{+}$be the $i$-th expanded special ramification scheme of type Cukierman. Then these ramification schemes are finite and satisfy, in the cycle group of $C \times C$ :

$$
\left[S W_{i}\right]=\left[D_{i}\right]+\left[E_{i}\right] \text { and }\left[E_{i}^{+}\right]=\left[E_{i}\right]+(g+1)\left[W_{i} \cap \Delta\right] .
$$

Proof. Since $C$ is general, by Statement 1 of Proposition 3.1, the set $S W_{i}$ is finite for each $i \geq 0$. Thus, so are $D_{i}$ and $E_{i}$ by Expression (16). It follows that $E_{i}^{+}$is finite, because $E_{i}^{+} \cap \Delta$ is the set of points $(P, P)$ such that $P$ is Weierstrass, whence is finite.

Recall the notation of Subsections 4.1, 4.2, 5.1 and 5.2, Set $r:=g+i-1$. Both equalities can be proved locally. Thus, let $(P, Q) \in C \times C$ and $\mathcal{O}$ be the local ring of $C \times C$ at $(P, Q)$. As a map of $\mathcal{O}$-modules, $\rho_{i, r+1}$ is given by a matrix $M$ of the form described in the proof of Lemma 5.3. Let us use the notation described in the statement of that lemma.

Let $K \subseteq \mathcal{O}$ define $S W_{i}^{+}$. Then $K=\left(z, z^{\prime}\right)$, where $z$ (resp. $\left.z^{\prime}\right)$ is the maximal minor obtained from $M$ by removing the last (resp. last but one) row. Notice that, from the nature of $M$ as a "wronskian matrix", $z^{\prime}$ is also the derivative of $z$ along $p_{1}$. Let $I$ and $J$ be the ideals of $\mathcal{O}$ defining $D_{i}^{+}$and $E_{i}^{+}$, respectively. Then $I$ and $J$ are the ideals of all the maximal minors of $M_{2}$ and $M$, respectively.

Now, since $C$ is a general curve, by Statement 2 of Proposition 3.1 ,

$$
h^{0}\left(\omega_{C}((i+1) P-(g+i) Q)\right) \leq 1 .
$$

This translates in the matrix obtained from $M_{1}$ by evaluating at $(P, Q)$ having rank at least $r$. Applying Lemma 5.3, there are $u, v \in \mathcal{O}$ such

$$
I=(z, u), \quad J=(z, v), \quad K=(z, u v) .
$$

Now, since $E_{i}^{+}$is finite-dimensional and $C \times C$ is smooth, the sequence $z, v$ is regular. The same holds for the sequence $z, u$ if $P \neq Q$. It follows that $\left[S W_{i}\right]=\left[D_{i}\right]+\left[E_{i}\right]$. 
The second equality in the statement of the proposition is obvious off $\Delta$. Thus, assume $Q=P$. Since $E_{i}^{+} \cap \Delta=W_{i} \cap \Delta$, we may also assume that $P$ is a Weierstrass point of $C$.

Let $t$ be a local parameter of $C$ at $P$, and $t_{1}, t_{2} \in \mathcal{O}$ be its pullbacks with respect to the projections $p_{1}$ and $p_{2}$. Then $t:=t_{2}-t_{1}$ is a local equation for $\Delta$. As we saw in Subsection [5.2, we have $z=t^{g} w$, where $w \in \mathcal{O}$ defines $W_{i}$, and is not divisible by $t$. Letting $\partial$ denote the derivative with respect to $t_{2}$, we have

$$
z^{\prime}=\partial z=\partial\left(t^{g} w\right)=g t^{g-1} w+t^{g} \partial w .
$$

Thus $t^{g-1}$ divides $z$ and $z^{\prime}$, and hence each element of $K$, in particular $u v$. Since $E_{i}^{+}$is finite, $t$ does not divide $v$, and hence $t^{g-1} \mid u$. Let $L:=t^{1-g} K$. Then there are two expressions for $L$ :

$$
L=\left(t w, u v / t^{g-1}\right) \quad \text { and } \quad L=(t w, g w+t \partial w) .
$$

Since $W_{i} \cap \Delta$ is finite, the sequences $g w+t \partial w, t$ and $w, t$ are regular. Thus, from the second expression for $L$ above, we get

$$
\ell(\mathcal{O} / L)=2 \ell(\mathcal{O} /(t, w))+\ell(\mathcal{O} /(w, \partial w))
$$

Now, $\ell(\mathcal{O} /(w, \partial w))=0$ because $w$ and $\partial w$ cut out $S W_{i}$, and $S W_{i}$ does not meet $\Delta$. Also, by Lemma 4.4, $W_{i}$ intersects $\Delta$ transversally. Thus $\ell(\mathcal{O} /(t, w))=1$, and hence $\ell(\mathcal{O} / L)=2$.

Now, since the sequence $z, v$ is regular, and $z=t^{g} w$, also the sequence $t w, v$ is regular. Thus, from the first expression for $L$ in (19), we get

$$
\ell(\mathcal{O} / L)=\ell\left(\mathcal{O} /\left(t w, u / t^{g-1}\right)\right)+\ell(\mathcal{O} /(t w, v))
$$

and whence $\ell(\mathcal{O} /(t w, v)) \leq 2$. Since $\mathcal{O}$ is regular, and the sequence $t w, v$ is regular, so is the sequence $v, w$. Thus

$$
\ell(\mathcal{O} /(t w, v))=\ell(\mathcal{O} /(t, v))+\ell(\mathcal{O} /(w, v))
$$

Since $E_{i}^{+}$contains $(P, P)$, the function $v$ is zero on $(P, P)$. Thus, since also $t$ and $w$ vanish on $(P, P)$, we get $\ell(\mathcal{O} /(t, v))=\ell(\mathcal{O} /(w, v))=1$. So, the multiplicity of $E_{i}^{+}$at $(P, P)$ is

$$
\ell(\mathcal{O} /(z, v))=g \ell(\mathcal{O} /(t, v))+\ell(\mathcal{O} /(w, v))=(g+1) .
$$

Since, by Lemma 4.4, the multiplicity of $W_{i} \cap \Delta$ at $(P, P)$ is 1 , we are done. 
5.5 Proposition. Let $C$ be a general smooth curve of genus $g \geq 1$ and $i$ a nonnegative integer. Let $S W_{i}$ be the $i$-th special ramification scheme of $C$. Then

$$
\int_{C \times C}\left[S W_{i}\right]=2 i g(g-1)\left((i+2)(g+i)^{2}+2(g+i)+2\right) .
$$

Proof. Recall the notation of Subsections 4.1, 4.2, 5.1 and 5.2. Since $C$ is general, $S W_{i}$ is finite. Also, $S W_{i}$ is the zero scheme of a section of the rank-2 bundle $\mathcal{J}_{p_{1}}^{1}\left(\mathcal{O}_{C \times C}\left(W_{i}\right)\right)$. Thus its class in the Chow group of $C \times C$ satisfies

$$
\left[S W_{i}\right]=c_{2}\left(\mathcal{J}_{p_{1}}^{1}\left(\mathcal{O}_{C \times C}\left(W_{i}\right)\right)\right)
$$

Using the truncation sequence for bundles of jets, we get

$$
\left[S W_{i}\right]=\left[W_{i}\right]\left(c_{1}\left(p_{2}^{*} \omega_{C}\right)+\left[W_{i}\right]\right)
$$

Now, $c_{1}\left(p_{2}^{*} \omega_{C}\right)=K_{2}$. Using Expression (11) for $j=i$, and taking into account that $K_{\ell}^{2}=0$ for $\ell=1,2$, we get

$$
\begin{aligned}
{\left[S W_{i}\right] } & =i(g+i+1)\left((g+i)^{2}+g+i+1\right) K_{2}[\Delta] \\
& +\frac{1}{2} i(i+1)\left((g+i)^{2}+g+i+1\right) K_{1} K_{2} \\
& +i^{2}(g+i+1)^{2}[\Delta]^{2}+i^{2}(g+i+1)(i+1) K_{1}[\Delta]
\end{aligned}
$$

Using Formulas (14) and (15), we get

$$
\begin{aligned}
\int_{C \times C}\left[S W_{i}\right] & =i(g+i+1)\left((g+i)^{2}+g+i+1\right)(2 g-2) \\
& +\frac{1}{2} i(i+1)\left((g+i)^{2}+g+i+1\right) 4(g-1)^{2} \\
& -i^{2}(g+i+1)^{2}(2 g-2)+i^{2}(g+i+1)(i+1)(2 g-2) .
\end{aligned}
$$

Simplifying, we get the claimed formula.

5.6 Theorem. Let $C$ be a general smooth curve of genus $g \geq 1$, and $i$ a nonnegative integer. Let $D_{i}$ and $E_{i}$ be the $i$-th special ramification schemes of type Diaz and Cukierman, respectively. Then $D_{i}$ and $E_{i}$ are reduced, and

$$
\int_{C \times C}\left[D_{i}\right]=g(g-1)\left((g+i-1)^{2}(i+1)^{2}-(g-1)^{2}\right)
$$

and

$$
\int_{C \times C}\left[E_{i}\right]=g(g-1)\left((g+i+1)^{2}(i+1)^{2}-(g+1)^{2}\right) .
$$


Proof. Recall the notation of Subsections 4.1, 4.2, 5.1 and 5.2, We will first compute the degrees of $D_{i}$ and $E_{i}$. First of all, since $E_{i}^{+}$is finite, and is the degeneracy scheme of $\rho_{i, g+i}$, applying Porteous formula ([8], Thm. 14.4, p. 254), we get the following expression for the class $\left[E_{i}^{+}\right]$in the Chow group of $C \times C$ :

$$
\left[E_{i}^{+}\right]=c_{2}\left(\mathcal{J}_{p_{1}}^{g+i}\left(\mathcal{L}_{i}\right)-p_{1}^{*} \mathcal{E}_{i}\right) .
$$

Now, $c_{2}\left(\mathcal{E}_{i}\right)=c_{1}\left(\mathcal{E}_{i}\right)^{2}=0$, since $C$ is one-dimensional. Thus

$$
\left[E_{i}^{+}\right]=c_{2}\left(\mathcal{J}_{p_{1}}^{g+i}\left(\mathcal{L}_{i}\right)\right)-c_{1}\left(\mathcal{J}_{p_{1}}^{g+i}\left(\mathcal{L}_{i}\right)\right) c_{1}\left(p_{1}^{*} \mathcal{E}_{i}\right) .
$$

Using the truncation sequence of the bundles of jets, we get

$$
\begin{aligned}
c_{1}\left(\mathcal{J}_{p_{1}}^{g+i}\left(\mathcal{L}_{i}\right)\right) & =\sum_{\ell=1}^{g+i+1}\left(\ell K_{2}+(i+1)[\Delta]\right) ; \\
c_{2}\left(\mathcal{J}_{p_{1}}^{g+i}\left(\mathcal{L}_{i}\right)\right) & =\sum_{m=2}^{g+i+1} \sum_{\ell=1}^{m-1}\left(\ell K_{2}+(i+1)[\Delta]\right)\left(m K_{2}+(i+1)[\Delta]\right) .
\end{aligned}
$$

Expanding, and using that $K_{2}^{2}=0$, we get

$$
\begin{aligned}
c_{1}\left(\mathcal{J}_{p_{1}}^{g+i}\left(\mathcal{L}_{i}\right)\right)= & \frac{1}{2}(g+i+1)(g+i+2) K_{2}+(i+1)(g+i+1)[\Delta] \\
c_{2}\left(\mathcal{J}_{p_{1}}^{g+i}\left(\mathcal{L}_{i}\right)\right)= & \frac{1}{2}(i+1)(g+i)(g+i+1)(g+i+2) K_{2}[\Delta] \\
& +\frac{1}{2}(i+1)^{2}(g+i)(g+i+1)[\Delta]^{2}
\end{aligned}
$$

Finally, using Formula (13) for $j=i$, and Formulas (14) and (15), we get

$$
\int_{C \times C}\left[E_{i}^{+}\right]=(i+1)^{2} g(g-1)(g+i+1)^{2} .
$$

Now, it follows from Proposition 4.4 that $W_{i}$ meets $\Delta$ transversally at $g^{3}-g$ points. Thus, using Proposition 5.4, we get

$$
\begin{aligned}
\int_{C \times C}\left[E_{i}\right] & =\int_{C \times C}\left[E_{i}^{+}\right]-(g+1)\left(g^{3}-g\right) \\
& =g(g-1)\left((g+i+1)^{2}(i+1)^{2}-(g+1)^{2}\right),
\end{aligned}
$$

the stated formula for the degree of $\left[E_{i}\right]$. 
Now, the expression for the degree of $\left[D_{i}\right]$ follows now from the equality $\left[S W_{i}\right]=\left[D_{i}\right]+\left[E_{i}\right]$ proved in Proposition 5.4 and Formula (20) for the degree of $\left[S W_{i}\right]$ proved in Proposition 5.5 .

Let us now show that $D_{i}$ and $E_{i}$ are reduced. Let $(P, Q) \in S W_{i}$. Let $\hat{\mathcal{O}}$ be the completion of the local ring of $C \times C$ at $(P, Q)$. Let $t_{1}$ and $t_{2}$ be local equations in $\hat{\mathcal{O}}$ for $\{P\} \times C$ and $C \times\{Q\}$, respectively. Then $\hat{\mathcal{O}}=\mathbb{C}\left[\left[t_{1}, t_{2}\right]\right]$. Let $w \in \mathcal{O}$ be a local equation for $W_{i}$. Since $(P, Q) \in S W_{i}$, and since $W_{i}$ is nonsingular by Proposition 4.4, we may assume that $w=t_{1}+u$, where $u \in \mathbb{C}\left[\left[t_{2}\right]\right]$. Now, let $w^{\prime}$ and $u^{\prime}$ be the derivatives of $w$ and $u$ with respect to $t_{2}$. Then the ideal defining $S W_{i}$ at $(P, Q)$ is $\left(w, w^{\prime}\right)$, and the multiplicity of the cycle $\left[S W_{i}\right]$ at $(P, Q)$ is $\ell\left(\hat{\mathcal{O}} /\left(w, w^{\prime}\right)\right)$. Notice that $w^{\prime}=u^{\prime}$, and

$$
\frac{\hat{\mathcal{O}}}{\left(w, w^{\prime}\right)} \cong \frac{\mathbb{C}\left[\left[t_{2}\right]\right]}{\left(u^{\prime}\right)}=\frac{\mathbb{C}\left[\left[t_{2}\right]\right]}{\left(u, u^{\prime}\right)} \cong \frac{\mathbb{C}\left[\left[t_{1}, t_{2}\right]\right]}{\left(t_{1}, w, w^{\prime}\right)}
$$

Thus the multiplicity of the cycle $\left[S W_{i}\right]$ at $(P, Q)$ is the multiplicity $m$ of $S W_{i} \cap(\{P\} \times C)$ at $(P, Q)$.

Since the formation of $S W_{i}$ commutes with base change, this multiplicity $m$ satisfies

$$
m=\mathrm{wt}_{V}(Q)-1
$$

where $V$ is the complete linear system of sections of $\omega_{C}((i+1) P)$. Now, by Propositions 3.1 and 3.4, the order sequence of $V$ at $Q$ satisfies

$$
\begin{aligned}
\epsilon_{j}(V, Q) & =j(j=0,1, \ldots, g+i-3), \\
\epsilon_{g+i-2}(V, Q) & \leq g+i-1, \\
\epsilon_{g+i-1}(V, Q) & \leq g+i+1 .
\end{aligned}
$$

Thus $m \leq 2$, with equality if and only if

$h^{0}\left(\omega_{C}((i+1) P-(g+i-1) Q)\right)=2 \quad$ and $\quad h^{0}\left(\omega_{C}((i+1) P-(g+i+1) Q)\right)=1$,

that is, if and only if $(P, Q) \in D_{i} \cap E_{i}$. Since $\left[S W_{i}\right]=\left[D_{i}\right]+\left[E_{i}\right]$ by Proposition 5.4 , it follows that $D_{i}$ and $E_{i}$ are reduced at $(P, Q)$.

\section{References}

[1] E. Arbarello, Weierstrass points and moduli of curves. Compo. Math. 29, no. 3, (1974), 325-342. 
[2] E. Arbarello, M. Cornalba, P. A. Griffiths and J. Harris, Geometry of algebraic curves. Grundlehren der mathematischen Wissenschaften, vol. 267, Springer, New York, 1985.

[3] CoCoATeam, CoCoA: a system for doing Computations in Commutative Algebra. Available at http://cocoa.dima.unige.it.

[4] F. Cukierman, Families of Weierstrass points. Duke Math. J. 58, no. 2, (1989), 317-346.

[5] C. Cumino, E. Esteves, and L. Gatto, Limits of special Weierstrass points. To appear.

[6] S. Diaz, Exceptional Weierstrass points and the divisor on moduli space that they define. Mem. Amer. Math. Soc., vol. 56, no. 327, Amer. Math. Soc., Providence, 1985.

[7] E. Esteves, Linear systems and ramification points on reducible nodal curves. In "Algebra Meeting" (A. Garcia, E. Esteves and A. Pacheco, Eds.), 21-35, Mat. Contemp., vol. 14, Soc. Bras. Mat., Rio de Janeiro, 1998.

[8] W. Fulton, Intersection theory. Grundlehren der mathematischen Wissenschaften, vol. 2, Springer, Berlin Heidelberg, 1984.

[9] L. Gatto and F. Ponza, Derivatives of wronskians with applications to families of Weierstrass points. Trans. Amer. Math. Soc. 351, no. 6, (1999), 2233-2255.

[10] J. Harris and D. Mumford, On the Kodaira dimension of the moduli space of curves. Invent. Math. 67 (1982), 23-86.

[11] S. Keel, Intersection theory of moduli space of stable n-pointed curves of genus zero. Trans. Amer. Math. Soc. 330 (1992), 545-574.

[12] F. Knudsen, The projectivity of the moduli space of stable curves, II. Math. Scand. 52 (1983), 161-199. 